\title{
DECOMPOSITIONS OF MODULES AND MATRICES
}

\author{
BY THOMAS S. SHORES AND ROGER WIEGAND ${ }^{1}$
}

Communicated by R. S. Pierce, May 7, 1973

\begin{abstract}
A canonical form for a module $M$ over a commutative ring $R$ is a decomposition $M \cong R / I_{1} \oplus \cdots \oplus R / I_{n}$, where the $I_{j}$ are ideals of $R$ and $I_{1} \subseteq \cdots \subseteq I_{n}$. A complete structure theory is developed for those rings for which every finitely generated module has a canonical form. The (possibly larger) class of rings, for which every finitely generated module is a direct sum of cyclics, is also considered, and partial results are obtained for rings with fewer than $2^{c}$ prime ideals. For example, if $R$ is countable and every finitely generated $R$-module is a direct sum of cyclics, then $R$ is a principal ideal ring. Finally, some topological criteria are given for Hermite rings and elementary divisor rings.
\end{abstract}

All rings in this announcement are commutative with 1, and all modules are unital. A canonical form for an $R$-module $M$ is a decomposition $M \cong R / I_{1} \oplus \cdots \oplus R / I_{n}$, where $I_{1} \cong \cdots \leqq I_{n} \neq R$. If $M$ has a canonical form, the ideals $I_{j}$ are uniquely determined $[\mathbf{K}]$. A CF-ring is a ring for which every finitely generated direct sum of cyclics has a canonical form. It can be shown that $R$ is CF if and only if

$$
R / I \oplus R / J \cong R /(I \cap J) \oplus R /(I+J)
$$

for every pair of ideals $I, J$.

By a valuation ring we shall mean a ring, possibly with zero-divisors, whose lattice of ideals is totally ordered. A ring $R$ is arithmetical, provided the local ring $R_{\mathrm{m}}$ is a valuation ring for each maximal ideal $\mathrm{m}$. Finally, an $h$-local domain [M1] is an integral domain such that (1) every nonzero ideal is contained in only finitely many maximal ideals, and (2) every nonzero prime ideal is contained in a unique maximal ideal.

THEOREM 1. Every CF-ring is a finite direct product of indecomposable $C F$-rings. The indecomposable $C F$-rings are precisely the rings $R$ such that (i) $R$ is arithmetical, (ii) $R$ has a unique minimal prime $P$, (iii) $R / P$ is an $h$-local domain, and (iv) every ideal contained in $P$ is comparable with every ideal of $R$.

Thus valuation rings and arithmetical $h$-local domains are CF-rings.

AMS (MOS) subject classifications (1970). Primary 13C05, 13F05; Secondary 15A21.

$K e y$ words and phrases. Canonical form, direct sum of cyclic modules, $h$-local domain, prime spectrum, elementary divisor ring, Hermite ring.

${ }^{1}$ The second author gratefully acknowledges support from the NSF for this research. 
The next theorem gives a fairly complete picture of the remaining indecomposable CF-rings.

THEOREM 2. Let $R$ be an indecomposable $C F$-ring with minimal prime $P$. Suppose $R$ is neither a valuation ring nor an h-local domain. Then $P^{2}=0$, $P$ is an indecomposable, torsion, divisible $R / P$-module, and $P=P_{m}$ for $a$ unique maximal ideal $\mathrm{m}$.

EXAMPLE. Let $A$ be an arithmetical $h$-local domain (for example a Dedekind domain) with more than one maximal ideal, and let $K$ be the quotient field of $A$. Let $\mathrm{m}$ be any maximal ideal of $A$, let $P=K / A_{\mathrm{m}}$, and make $R=A \oplus P$ into a ring by setting $(a, p)\left(a^{\prime}, p^{\prime}\right)=\left(a a^{\prime}, a p^{\prime}+a^{\prime} p\right)$. Then $R$ is a CF-ring which is neither a domain nor a valuation ring.

Let us define an FGC-ring to be a ring $R$ such that every finitely generated $R$-module is a direct sum of cyclic modules. If, in addition, $R$ is CF, we say $R$ is an FGCF-ring. The local FGC-rings have been characterized as the almost maximal valuation rings [G]. Thus, if $R$ is FGC, it follows that $R_{\mathrm{m}}$ is almost maximal for every $\mathrm{m}$, and it is not hard to see that $R$ is Bezout, that is, every finitely generated ideal of $R$ is principal. In general, these two conditions are far from sufficient. Surprisingly, CF-rings have the requisite "finiteness" conditions:

THEOREM 3. A CF-ring $R$ is FGCF if and only if $R$ is Bezout and $R_{\mathrm{m}}$ is almost maximal for each maximal ideal $\mathrm{m}$.

If, in the example above, $A_{\mathrm{m}}$ is maximal and all the other localizations of $A$ are almost maximal, the $\operatorname{ring} R=A \oplus P$ can be shown to have almost maximal localizations. If, in addition, $A$ is Bezout (for example, a ring of type $\mathrm{I}$ [M2]) then $R$ is an FGCF-ring.

A direct proof of Theorem 3 would appear to be difficult. Theorems 1 and 2, however, reduce the task to consideration of the three types of indecomposable CF-rings. Only the third type presents any difficulty, and it is handled by techniques similar to those in [G].

The problem of classifying FGC-rings seems to be much harder. It would be a great help to know that an FGC-ring has only finitely many minimal primes. (Indeed, this was a major step in the characterization of CF-rings.) We have some partial results in this direction:

THEOREM 4. Let $R$ be an FGC-ring. Then every compact set of minimal primes is finite. If $R$ has fewer than $2^{c}$ prime ideals, then $R$ has only finitely many minimal primes.

COROLLARY. If $R$ is an FGC-ring with fewer than $2^{c}$ prime ideals then $R$ has noetherian maximal ideal space.

COROLlary. Every countable FGC-ring is a principal ideal ring. 
To prove Theorem 4, one uses methods similar to those in $[\mathbf{P}]$ to show that a fairly benign condition on $\operatorname{spec}(R)$, the prime spectrum of $R$, prevents FGC. The statement of this condition involves two topologies on $\operatorname{spec}(R)$ - the usual (Zariski) topology, and the patch topology, which is generated by declaring that the quasicompact open sets in the usual topology shall be both open and closed.

THEOREM 5. Suppose $\operatorname{spec}(R)$ has a point in the patch closure of each of three pairwise disjoint Zariski open sets. Then $R$ is not an FGC-ring.

We know of no examples of FGC-rings which are not already FGCF. Nonetheless it seems plausible that a semilocal, locally almost maximal domain might have FGC without being $h$-local.

When we restrict our attention to decompositions of finitely presented modules, we are led inevitably to questions about diagonalization of matrices. A module $M$ with $m$ generators and $n$ relations is isomorphic to $R^{m} / K$, where $K$ is generated by the columns of an $m \times n$ matrix $A$. In this case we say $M$ is presented by $A$. It is well known that if $A$ and $B$ are equivalent then they present isomorphic modules, but that the converse fails, even if $A$ and $B$ have the same size. It has recently been shown, however, that if every finitely presented $R$-module is a direct sum of cyclics then every matrix over $R$ is equivalent to a diagonal matrix [LLS].

The situation is somewhat different for canonical forms. We say a matrix $A$ has a canonical form provided $A$ is equivalent to a diagonal matrix $\left[d_{i j}\right]$ in which $d_{i+1, i+1}$ divides $d_{i, i}$ for all $i$. In [LLS] it was proved that $R$ is Bezout if and only if every diagonal matrix over $R$ has a canonical form. On the other hand, $R$ is arithmetical if and only if every finitely presented direct sum of cyclics has a canonical form.

An elementary divisor ring is a ring for which every matrix has a canonical form. (By [LLS] it is enough to check that every finitely presented module is a direct sum of cyclics.) In the standard examples of elementary divisor rings (for example, the adequate rings [LLS]), the dimension of the maximal ideal space is at most one. The following theorem, and a construction due to Heinzer $[\mathbf{H}]$, show that higherdimensional examples exist.

THEOREM 6. Every Bezout ring with noetherian maximal ideal space is an elementary divisor ring.

Finally, we turn our attention to an intermediate class of rings, between Bezout rings and elementary divisor rings. A ring $R$ is Hermite provided every matrix is equivalent to a triangular matrix. (Equivalently, every 1 by 2 matrix is equivalent to a diagonal matrix [K].) $\mathbf{M}$. Henriksen has raised the following question: If $R$ is a Bezout ring with compact minimal prime spectrum, is $R$ Hermite? While we suspect that the answer is "no", 
the following results indicate that a counterexample would have to have a rather perverse nilradical.

THEOREM 7. Let $R$ be a Bezout ring with compact minimal prime spectrum. Then $R$ is Hermite if and only if every 1 by 2 matrix with nilpotent entries is equivalent to a diagonal matrix.

COROllary. Let $R$ be a Bezout ring with compact minimal prime spectrum. If the nilradical of $R$ is T-nilpotent, then $R$ is Hermite.

\section{REFERENCES}

[G] D. T. Gill, Almost maximal valuation rings, J. London Math. Soc. (2) 4 (1971), $140-146$.

[H] W. Heinzer, J-noetherian integral domains with 1 in the stable range, Proc. Amer. Math. Soc. 19 (1968), 1369-1372. MR 38 \#145.

[K] I. Kaplansky, Elementary divisors and modules, Trans. Amer. Math. Soc. 66 (1949), 464-491. MR 11, 155.

[LLS] M. D. Larsen, W. J. Lewis and T. S. Shores, Elementary divisor rings and finitely presented modules, Trans. Amer. Math. Soc., (to appear).

[M1] E. Matlis, Decomposable modules, Trans. Amer. Math. Soc. 125 (1966), 147-179. MR 34 \# 1349.

[M2] - Rings of type I, J. Algebra 23 (1972), 76-87.

[P] R. S. Pierce, Modules over commutative regular rings, Mem. Amer. Math. Soc. No. 70 (1967). MR 36 \#151.

DePartment of Mathematics, University of Nebraska, Lincoln, Nebraska 68503. 